\title{
78. The Determination of the Thickness of an Adsorbed Molecular Layer of Water.
}

\author{
By Yosioki IsIKawa. \\ (Comm. by Y. OSAKA, M.t.A., July 12, 1943.)
}

Numerous studies ${ }^{1)}$ have been made concerning the adsorption of a gas upon a solid surface. From all of them is drawn the conclusion that the fundamental assumption in the Langmuir theory ${ }^{2)}$ that the adsorption layer is only one molecule thick has established its truth except under special conditions. On the other hand, the multi-molecular layer theory ${ }^{3)}$ can not be denied. The question whether van der Waal's adsorption or any kind of adsorption on solid ceases when the surface is covered by a layer one molecule or one atom thick, does not yet appear to have been answered unequivocally. The principal difficulty lies in estimating the surface area of the solid. Avoiding the difficulty as to area determination, there are the following methods of deducing the thickness of the adsorption layer: the measurements of the accommodation coefficient, of the thermionic emission, of the elliptical polarisation of light reflected from the surface, and of the surface potential.

By measuring the desorption of the adsorbed water molecules by slow electron impact, the present author succeeded in deducing the thickness of the adsorption layer of the water molecules without measuring the area. He established that the adsorption of the water molecules upon a platinum plate is in the form of a mono-molecular-tri-molecular at the highest-layer under very low pressures, except the case of the pressure of the adsorbate approximating to the saturated vapour pressure, when the formation of thicker layers and even condensation in bulk may occur. The result obtained by this method is in fairly good agreement with those obtained by the above mentioned indirect methods.

\section{Experimental Results.}

The apparatus and procedure used are the same as given in the preceding papers ${ }^{4}$.

1) Outgassing.

Very careful outgassing of the glass is necessary in this work, since the adsorptive capacity of the glass vessel is usually far larger than that of the platinum surface used.

1) McBain, The Sorption of Gases and Vauuurs by Solids. (Routledge, 1932). Berkman, Catalysis (Reinhold, 1940).

2) I. Langmuir, J. Am. Chem. Soc., 38 (1916), 2221.

3) s. Brunauer, P. H. Emmett, and E. Teller, J. Am. Chem. Soc., 60 (1938), 309. $\therefore$ Brunatuer, L.S. Deming, W.E. Deming and E. Teller, J. Am. Chem. Soc., 62 (19.10), 1723.

4) Y. Isikaıva, Proc., 18 (1942), 246, $390 ; 19$ (1943), 380. 
The vessel wall of the diode and the platinum plate were outgassed at $350^{\circ} \mathrm{C}$ for several hours, especially the latter was red-heated by electron bombardment to expel the absorbed and adsorbed gases. Being cooled at room temperature the diode was kept from the contamination of any other vapour with a trap immersed in liquid air. This treatment afforded such a very desirable outgassing condition as the desorption of gas was hardly caused by slow electron impact.

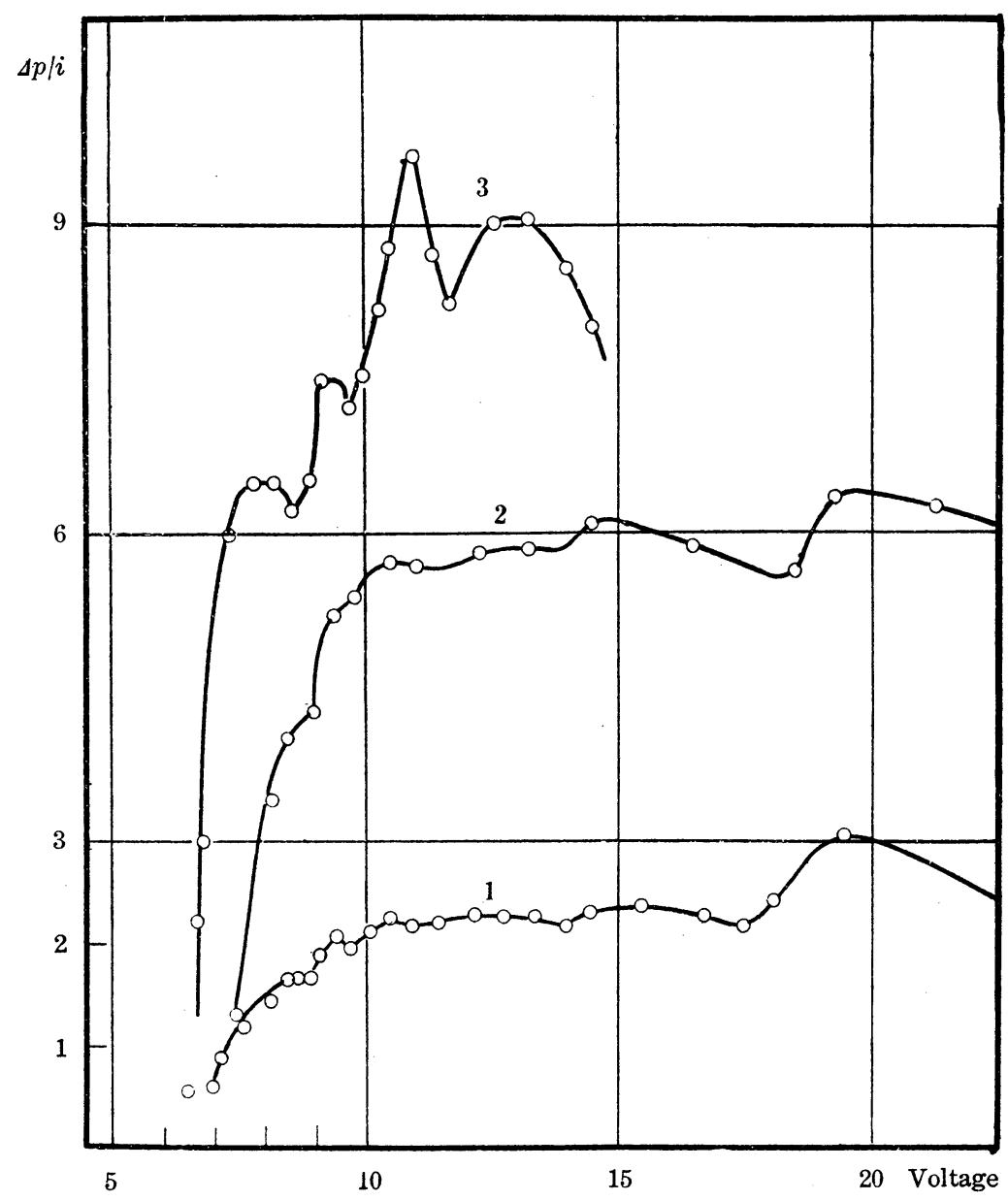

Fig. 1. The desorption curves of the multimolecular layer of adsorbed water by electron impact. (the curves 1,2,3 in Fig. represent mono-, di- and tri-molecular layer respectively.)

2) Adsorption of water molecules on the platinum plate.

When a trace of water is introduced and vaporized during the course of pumping, the water molecules cover the platinum plate and the glass wall in a thick molecular layer. Further pumping, however, causes the desorption. The process of such a desorption is known 
from the measurement of the volume of the decomposed water molecules by the heated oxide-coated filament. Such a large vol of the permanent gas as immeasurable by means of the Pirani-gauge is desorbed at first. The amount of the desorbed gas is decreased rapidly for 2 hours, and then gradually. After the outgassing of several hours more, a series of impact experiments were performed on the platinum plate and the glass wall was maintained at room temperature in the state of the smallest possible amount of the gas to be desorbed.

3) The characteristics of the desorption curve.

The experimental results are shown as the desorption curve in Fig. 1, where accelerating potential in V. is taken as the abscissa. This potential is the corrected value obtained by reducing the $1.5 \mathrm{~V}$. of the contact potential difference from the applied voltages. $\Delta p / i$ is taken as the ordinate; $s p$ being the deflection of the galvanometer in

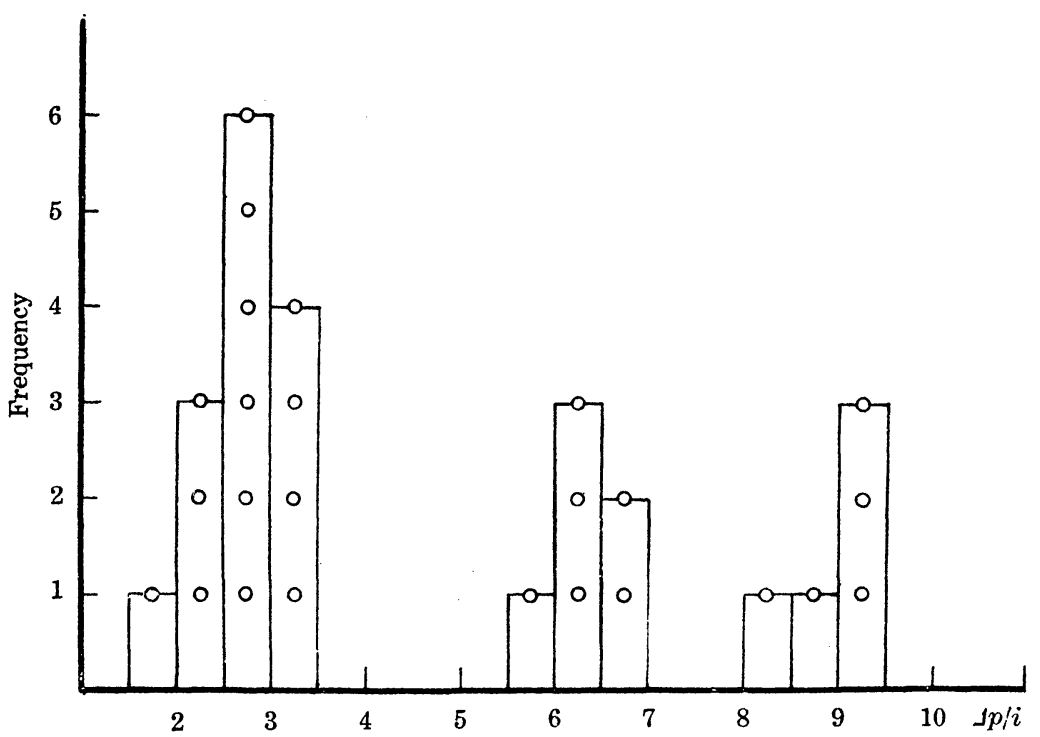

Fig. 2. Values $\Delta p / i$ and their freque

cm., denotes the amount of the desorbed gas for 3 minutes; $i$ denotes the impinging electron current in $\mathrm{mA}$. As seen in the figure, three desorption curves, different in height, are very similar in appearance. From the 26 desorption curves obtained, $\Delta p / i$ values were measured in the flat range from 10.5 to $15 \mathrm{~V}$. The relation between the values thus obtained and the frequency of their occurrence is shown in Fig. 2. In the figure, the value of $\Delta p / i$ is taken as the abscissa, being shown at an interval of 0.5 and the frequency as ordinate. The values of $\Delta p / i$ fall in the neighbourhood of 3,6 , and 9 without showing a continuous distribution.

In the preceding experiment, the adsorption layer of water was obtained in consequence of pumping. But the adsorption layer could be also rapidly outgassed by moderate heating. 
The author examined how $\Delta p / i$ value decreased by the prolonged impact of the slow electrons at a fixed voltage upon the adsorbed layer. A slow electron impact was continued several hours with an electron current $0.3 \mathrm{~mA}$. and accelerating voltage $11 \mathrm{~V}$. Thus, the value of $\Delta p / i$ is taken as the ordinate and the time elapsing after the electron impact as the abscissa. Two hours after the impact $\Delta p / i$ varies suddenly from 9 to 6 and then remains unchanged for more than 2 hours, as seen in Fig. 3.

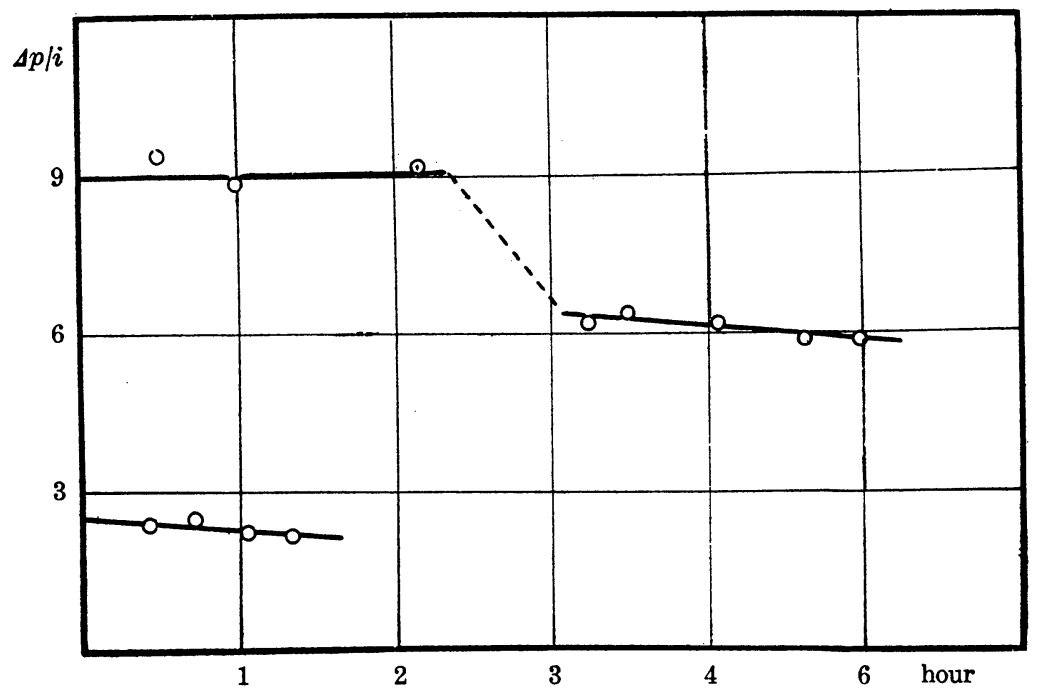

Fig. 3. The variation of $\Delta p / i$ by the impact of electrons at the definite voltage.

4) The influence of the adsorbed water molecule film of a diode upon that of a platinum plate.

(a). The effect of the heat treatment of the diode.

A thick adsorption layer is formed on the wall and the platinum surface in consequence of evaporation of the water introduced in the diode. If adequate heating and pumping as well as mere pumping is made for the layer, the states of adsorption corresponding to the curves, (1), (2) and (3), in Fig. 1 are established in a short time. Heating and pumping for about 40 minutes at $160-220^{\circ} \mathrm{C}$, and about $220^{\circ} \mathrm{C}$ produce the states corresponding to the curves (3) and (2) in Fig. 1 respectively. When an electron current $5 \mathrm{~mA}$. accelerated to $300 \mathrm{~V}$. is allowed to impact on the platinum plate for about 3 hours and the diode is heated to about $200^{\circ} \mathrm{C}$, such a poor adsorbed state of water as the value of $\Delta p / i$ is less than 1 at about $12 \mathrm{~V}$. is also established.

(b). The freezing of the adsorbed water molecules on the surface of a diode.

The immersion of the wall of a diode in liquid air causes the decrease in the value of $\Delta p / i$ every time when an experiment is carried 
out. This is shown in Fig. 4. In this case, a desorption curve as given in Fig. 1, can not be obtained.

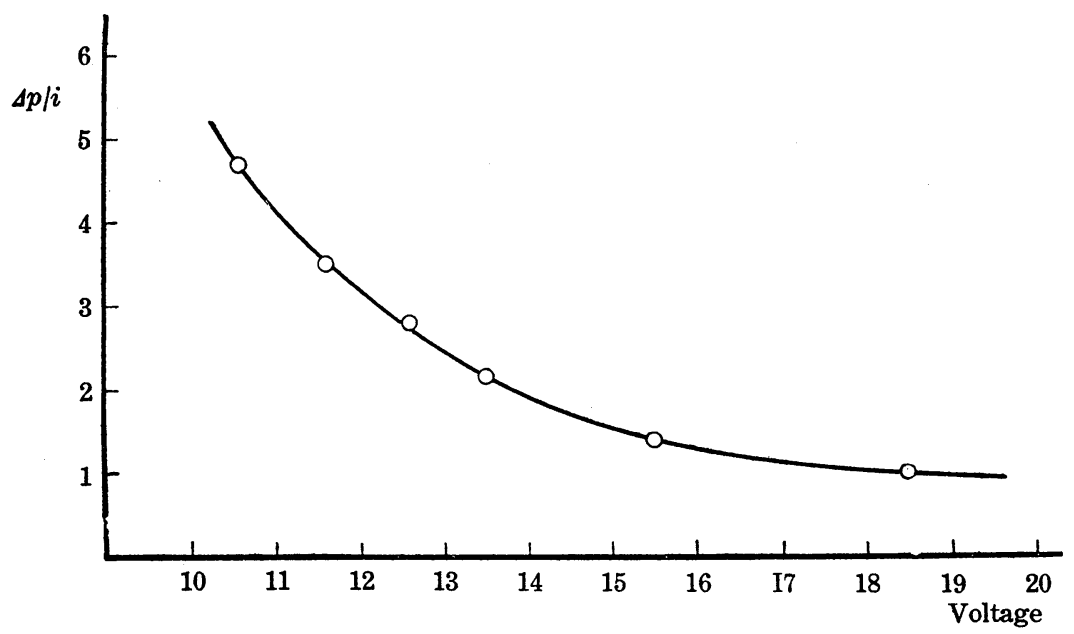

Fig. 4. The desorption curve of the adsorbed layer of water (the diode is immersed in liquid air).

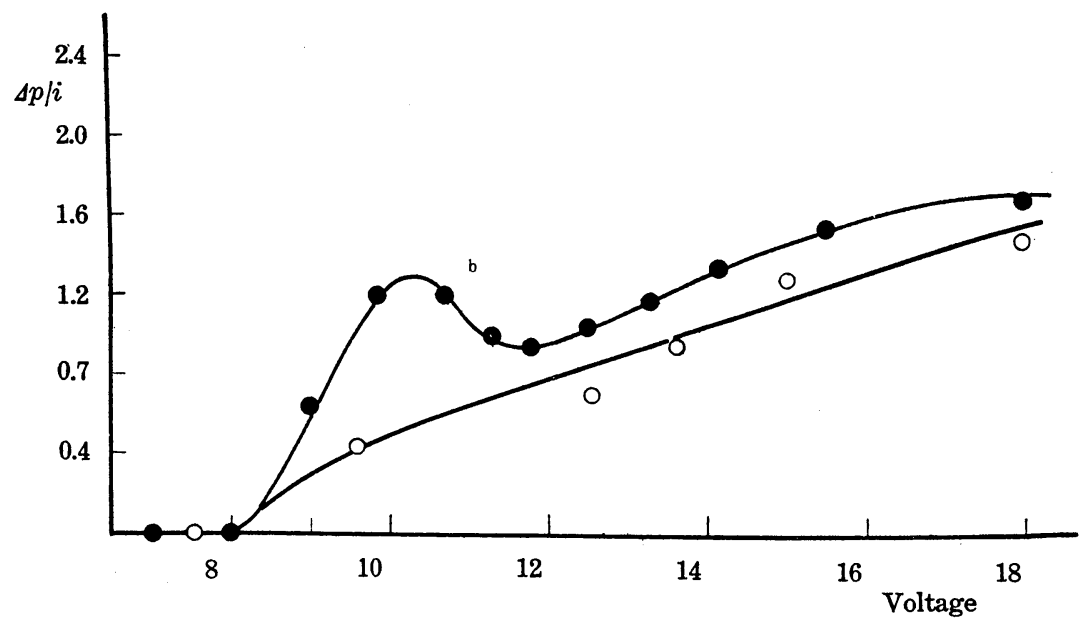

Fig. 5. The desorption curve of the co-adsorbed film of water and hydrogen molecules by electron impact.

- the desorption curve of the co-adsorbed film.

o the desorption curve of the adsorbed water molecule.

5) Simultaneous adsorption of hydrogen molecules on water covered surface of a platinum plate.

Each adsorbed layer of water corresponding to the curves in Fig. 1 was left to stand for several hours after introducing hydrogen at $3 \mathrm{~cm} . \mathrm{Hg}$ and then pumped ; the experiment on slow electron impact was then performed to determine the change of the desorption curve. 
The curves corresponding to (1), (2) and (3) in Fig. 1 show no change in spite of the introduction of hydrogen. But in the case where the value of $\Delta p / i$ is about 1 , a maximum characteristic to hydrogen molecule ${ }^{4}$ appears at about $10 \mathrm{~V}$. This indicates that there exist simultaneously both water and hydrogen molecules in the adsorbed layer. In Fig. 5, (a) is the desorption curve of the adsorbed water nolecules in the case when hydrogen is not introduced yet; (b) is that in the case when hydrogen has been introduced.

\section{Discussion.}

In the preceding report ${ }^{5)}$, the author discussed extensively the mechanism of desorption of the adsorbed water molecules by electron impact, and made clear that the desorption phenomenon is due to the dissociation or predissociation of the molecules by electron impact.

The number of the collisions between the adsorbed molecules and electrons, which cause such dissociation and predissociation is proportional to a fraction of the surface covered with the adsorbed molecules $c$ and the electron current $i$. Accordingly the desorbed amount can be expressed thus :

$$
\Delta p=\varepsilon \cdot c \cdot i
$$

where $\varepsilon$ is the probability of dissociation by an electron impact. $\Delta p / i$ : the height of the desorption curve is equal to the product of the dissociation probability $\varepsilon$ and the adsorbed fraction $c$. In Fig. 1, however, the three desorption curves are found to differ in height, and in Fig. 2, the height, $\Delta p / i$, is seen in such a multiple relation as 3,6 and 9.

Such discontinuity of the value of $\Delta p / i$ is due to that of the fraction of the covered surface $c$ proportional to $\Delta p / i$. On a metal surface where the same atoms, though with some irregularity, are arranged uniformly and two-dimensionally, it is not admitted that a mono-molecular layer is discontinuously built up by the successively adsorbed molecules. The cause of such discontinuity is to be sought in the multiplicity of the structure of the adsorption layer.

The curves, (1), (2) and (3) in Fig. 1 are considered to correspond to the mono-molecular layer, di-molecular one, and tri-molecular one respectively.

As for such multi-molecular layers. let the fractions of the adsorbed surfaces of the first, the second and the third layers be represented by $c_{1}, c_{2}$ and $c_{3}$ respectively. Suppose that the electron current $i$, after bombarding the layers and passing through them, reaches the interior of the metal, and the desorption efficiencies of the impinging electron for each layer will be calculated as follows:

For the third layer $\Delta p_{3}=\varepsilon_{3} c_{3} i$.

For the second layer $\Delta p_{2}=\varepsilon_{2} c_{2}\left(i-\varepsilon_{3} c_{3} i\right)$

For the first layer $\Delta p_{1}=\varepsilon_{1} c_{1}\left\{i-\varepsilon_{3} c_{3} i-\varepsilon_{2} c_{2}\left(i-\varepsilon_{3} c_{3} i\right)\right\}$.

5) Y. Isikawa, Proc., 19 (1943), 380. 
(The suffixes 1, 2 and 3 have the relation to the first, the second and the third layer respectively.) The preceding experiment $t^{5)}$ has proved that the electronic state of the adsorbed water molecules is hardly different from that of the homogeneous phase. Accordingly, it is presumed that the dissociation probability of the adsorbed molecules are not much different from those of the homogeneous phase. Hence $\varepsilon_{1}=\varepsilon_{2}=\varepsilon_{3}$. In the case of curve (1) in Fig. 1, if $c_{1}$ is taken to be $1, \varepsilon_{1}$ will be $4 \times 10^{-4}$. As the high power terms of $\varepsilon$ can be neglected, the ratio of the desorption efficiency $\Delta p / i$ of electrons concerning these three mono-, di- and tri- molecular layers is as follows :

$$
\begin{aligned}
\left(\frac{\Delta p}{i}\right)_{\text {mono }}:\left(\frac{\Delta p}{i}\right)_{\mathrm{di}}:\left(\frac{\Delta p}{i}\right)_{\text {tri }}= & \varepsilon_{1} c_{1}:\left(\varepsilon_{1} c_{1}+\varepsilon_{2} c_{2}\right):\left(\varepsilon_{1} c_{1}+\varepsilon_{2} c_{2}+\varepsilon_{3} c_{3}\right) . \\
& =3: 6: 9 .
\end{aligned}
$$

On calculation, we obtain $c_{1}=c_{2}=c_{3}$. This shows that the adsorbed surface fraction of each layer of the multi-molecular layers formed are quite the same. Such arrangement of molecules requires a fully packed adsorption layer: namely such an adsorption film has scarcely any attachment and hole. This has been also kinetically discussed ${ }^{6)}$. The stableness of those complete di-molecular and tri-molecular layers is confirmed from the fact that the value of $\Delta p / i$ is kept constant in the process of many hours' impact, though it changes from 9 to 6 in relatively short time.

When a mono-molecular layer has been fermed, a further heating and pumping produces an uncovered part on the platinum surface. For example, after the adsorption layer of water formed has been heated and pumped above $250^{\circ} \mathrm{C}$ for a long time, as seen in Fig. 5, the introduction of hydrogen changes it into an adsorption layer where the molecules of both hydrogen and water exist simultaneously. This formation is possible only in the case when $\Delta p / i$ is smaller than 3. As the value of $\Delta p / i$ becomes larger from 0 to 3 , the part yet uncovered diminishes in dimension; when the value reaches 3 , there exists a mono-molecular film of the water molecules only, hydrogen being no more adsorbed. Similarly, any simultaneous adsorption of hydrogen with water is not observed on the di-molecular and the tri-molecular layers. It is proved that the covered fractions $c_{1}, c_{2}, c_{3}$ of each layer are 1 equally, that is, the surface is covered completely with water molecules.

These adsorption films on such a metal surface have co-relationship with the adsorption layer of water formed on the wall of the diode.

In spite of the desorption quantity caused by more than 2 hours' electron impact which corresponds to several square centimeters mono-molecular film by calculation, the value of $\Delta p / i$ remains unchangea as seen in Fig. 3 and the film on the metal is not decreased in thickness. Nevertheless, heating and pumping treatment of the wall of the diode above $200^{\circ} \mathrm{C}$ instantly decreases the value of $\Delta p / i$ below

6) M. Volmer, Kinetik der Phasenbildung, 32, (Steinkopff, 1939). 
2 and it leads to the decrease in thickness of the layer. It is considered that as soon as the adsorbed molecules on the metal are desorbed in consequence of electron impact, these are probably made up by the molecules adsorbed on the diode wall. When the adsorption layer of water on the wall is frozen with liquid air, the value of $\Delta p / i$, as seen in Fig. 4, becomes smaller every time when electron impact is performed, and the constancy of the value of $\Delta p / i$ as in Fig. 3 is not so long maintained. This fact indicates that there exists an adsorption equilibrium established between the adsorbed films on the metal and the wall.

In conclusion the author has great pleasure in expressing his sincere thanks to Professor.S. Horiba for his continued guidance throughout this research.

Acknowledgments are expressed to the Imperial Academy for a grant in aid of the present work. 